\title{
Sirtuin 7 promotes colorectal carcinoma proliferation and invasion through the inhibition of E-cadherin
}

\author{
ZHIGANG DENG, XINGBIAO WANG, XUAN LONG, WANZHONG LIU, \\ CHUNHUA XIANG, FENG BAO and DONG WANG \\ Department of General Surgery, Mianyang Central Hospital, Mianyang, Sichuan 621000, P.R. China
}

Received August 19, 2016; Accepted December 11, 2017

DOI: $10.3892 /$ etm.2017.5673

\begin{abstract}
Sirtuin 7 (Sirt7) is a member of the sirtuin protein family and is implicated in various carcinomas; however, the function of Sirt7 in colorectal carcinoma (CRC) remains unclear. The present study aimed to explore the biological function of Sirt7 in CRC tissues and cell lines, and to investigate the potential underlying mechanism by performing reverse transcription-quantitative polymerase chain reaction analyses, western blot analyses, luciferase reporter assays, cell proliferation and invasion assays. It was demonstrated that Sirt7 presented a higher expression in CRC tissues and cell lines compared with that in normal tissues and cells, and this higher expression was correlated with the tumor size, the tumor, node and metastasis stage and distant metastasis. Knockdown of Sirt7 repressed the proliferation ability of SW620 and HCT116 cells in vitro, while ectopic expression of Sirt7 increased the epithelial-mesenchymal transition and invasion in HT29 and SW480 cells. Notably, these functional effects of Sirt7 were exerted through the repression of E-cadherin. Thus, the data of the present study indicated a novel mechanistic role for Sirt7 as an oncogene in CRC malignancy, and Sirt7 may be a potential therapeutic target.
\end{abstract}

\section{Introduction}

The sirtuin (Sirt) protein family belongs to the class III of NAD-dependent histone deacetylases and comprises seven members (termed Sirt1-7) (1). Sirt proteins have been widely investigated for their deacetylation activities, characteristic by deacetylating histones, such as $\mathrm{H} 3$, and non-histone proteins, such as cluster of differentiation (CD)K9; Sirt proteins are frequently overexpressed in several types of cancer (2). Sirt7 is a histone $\mathrm{H} 3$ on lysine 18 (H3K18) deacetylase and, as a

Correspondence to: Dr Zhigang Deng, Department of General Surgery, Mianyang Central Hospital, 12 Changjia Alley, Jingzhong Street, Mianyang, Sichuan 621000, P.R. China

E-mail: chargedgon@gmail.com

Key words: sirtuin 7, proliferation, metastasis, colorectal carcinoma, E-cadherin new member of this family, has been reported to be mainly localized in the nucleus (3). In addition, Sirt7 has been reported by different researchers to be involved in certain carcinomas, including ovarian (4), gastric (5), breast (6) and cervical cancer (7). However, the role and function of Sirt7 in colorectal carcinoma (CRC) remains to be investigated. As Sirt1 serves a role in the promotion of epithelial-mesenchymal transition and metastasis in colorectal cancer the function of Sirt7 in CRC was explored.

CRC is the third most common malignancy worldwide and the fourth leading cause of cancer-associated mortalities, with rectal carcinoma constituting $28 \%$ of all CRC cases $(8,9)$. Despite the advances in surgery, chemotherapy and radiotherapy in the past decades, various clinical side effects occur in these traditional treatments $(10,11)$. Furthermore, distant metastasis, particularly liver metastasis, is the main cause of mortality in patients with CRC, and the current therapy is largely unsuccessful due to tumor resistance $(12,13)$. Therefore, improving the understanding of the tumorigenesis process and the molecular mechanism underlying CRC is of great importance for developing novel diagnostic and therapeutic strategies. The present study aimed to explore the expression and function of Sirt7 in CRC, in order to bring novel insight into understanding the mechanisms about CRC.

\section{Materials and methods}

Patients. A total of 60 fresh tumor tissues and their adjacent non-tumorous tissues were obtained from patients who were diagnosed with CRC and underwent surgery at Mianyang Central Hospital (Mianyang, China) between January 2009 and December 2009. Patients subjected to chemotherapy prior to surgery were excluded from the current study. The tissues were obtained immediately after surgery and frozen at $-196^{\circ} \mathrm{C}$ in liquid nitrogen until further use. Written informed consent was obtained from each patient at the day of surgery, and the study was approved by the local Research Ethics Committee of Mianyang Central Hospital. The clinicopathological characteristics of patients are listed in Table I.

The tumor stage was defined using the seventh edition of the tumor, node and metastasis (TNM) classification for CRC of the American Joint Committee Cancer (14). Furthermore, lymph node metastasis was identified using the lymph node pathological examination during the surgery, and the distant metastasis 
was identified using abdominal and pulmonary computed tomography prior to surgery. The diagnosis of all patients was histopathologically confirmed. All the patients were followed up to generate the overall survival times, with a total follow-up period of 6 years (range, 1-6 years). The follow-up information of all the participants was updated every 3 months by a telephone conversation and questionnaire letters. The survival times were calculated from the surgery date to the recurrence or metastasis-associated mortality. Patients were divided into two groups for assessing overall survival according to their median Sirt7 mRNA expression levels, specifically the high and low Sirt7 expression groups. Sirt7 mRNA expression in frozen $\mathrm{CRC}$ tumor tissues and paired adjacent non-tumor tissues was measured by reverse transcription-quantitative polymerase chain reaction (RT-qPCR).

Cell culture. Human SW620 (CCL-227 ${ }^{\mathrm{TM}}$ ) and SW480 (CCL-228 ${ }^{\mathrm{TM}}$ ) colon carcinoma cells were purchased from American Type Culture Collection (ATCC; Manassas, VA, USA). In addition, HCT116 and HT29 colon cancer cell lines, as well as human normal colon epithelium FHC cell line, were purchased from Shanghai Bioleaf Biotech Co., Ltd. (Shanghai, China). The aforementioned cells were cultured in RPMI 1640 medium (Hyclone; GE Healthcare Life Sciences, Logan, UT, USA) containing 10\% fetal bovine serum (Sigma-Aldrich; Merck KGaA, Darmstadt, Germany) in a $5 \% \mathrm{CO}_{2}$ atmosphere at $37^{\circ} \mathrm{C}$.

Reagents and transfection. Sirt7 siRNAs were obtained from Sigma-Aldrich; Merck KGaA. The sequence of the siRNA against Sirt7 (siRNA ID, SASI Hs01_00133900) was 5'-CGC CAAATACTTGGTCGTCTA-3' and CDH1 (E-cadherin; siRNA ID, SASI Hs01_00086310) was 5'-GAGATTGCACCG GTCGACAAAGCTC-3', and the control siRNA sequence [scramble control RNA (SCR)] was 5'-CCTAAGGTTAAG TCGCCCTCG-3'. A final concentration of $50 \mathrm{nM}$ Sirt7, $50 \mathrm{nM}$ E-cadherin or $50 \mathrm{nM}$ of their corresponding negative control siRNA was used for transient transfection with Lipofectamine 2000 (50 $\mu$; Invitrogen; Thermo Fisher Scientific, Inc., Waltham, MA, USA) at a ratio of 1:1, and the solutions were incubated for $20 \mathrm{~min}$ at room temperature. Subsequently, the siRNA mixture was added to the cells and incubated for $8 \mathrm{~h}$ at $37^{\circ} \mathrm{C}$, according to the manufacturer's protocol.

The Sirt7 full-length sequence was amplified from human genomic DNA and cloned into the lentiviral vector GV208 using the EcoRI and NotI sites (Genchem, Shanghai, China), obtaining pGV-Sirt7. The primers for Sirt7 were as follows: 5'-ATATGAATTCGCCACCATGGCAGCCGG GGGTCTGATC-3' (forward) and 5'-ATAAGGATGCGG CCGCTTACGTCACTTTCTTCCTTTTTGT-3' (reverse). The E-cadherin lentiviral expression vector was purchased from Genchem.

293 T cells (CRL-3216 ${ }^{\mathrm{TM}}$; ATCC), used for virus packaging, were cultured in Dulbecco's modified Eagle's medium in a $37^{\circ} \mathrm{C}$ incubator with $5 \% \mathrm{CO}_{2}$. pGV-Sirt7, pHelper 1.0 and pHelper 2.0 were cotransfected into $293 \mathrm{~T}$ cells using Lipofectamine ${ }^{\circledR} 2000$. At $8 \mathrm{~h}$ after transfection, the medium was refreshed, and at $48 \mathrm{~h}$ after transfection, the $25 \mathrm{ml}$ supernatant was harvested by centrifugation at $12,000 \mathrm{x}$ g for $15 \mathrm{~min}$ at $4^{\circ} \mathrm{C}$ for further use. Empty lentiviral GV208 vector was used as the negative control (pGV-NC). The SW620 and SW480 CRC cells were infected with either pGV-Sirt7 or pGV-NC in the presence of $5 \mu \mathrm{g} / \mathrm{ml}$ Polybrene (Sigma-Aldrich; Merck $\mathrm{KGaA}$ ). At $72 \mathrm{~h}$ post-infection, the efficiency of infection was measured by performing RT-qPCR. The E-cadherin lentiviral expression vector was purchased from Genchem.

RNA isolation and RT-qPCR. Total RNA was isolated from the aforementioned cell lines and tissues using TRIzol reagent (Invitrogen; Thermo Fisher Scientific, Inc.) according to the manufacturer's protocol. The purification and quantity of the RNA was measured with a NanoDrop 2000 spectrophotometer (Thermo Fisher Scientific, Inc.). Next, $1 \mu \mathrm{g}$ RNA was reverse-transcribed into cDNA using the TransGen First Strand cDNA Synthesis kit (TransGen Biotech, Beijing, China). qPCR was then performed using SYBR-Green Master Mix (Roche Applied Science, Penzberg, Germany) on an Applied Biosystems Prism 7500 detection system (Applied Biosystems; Thermo Fisher Scientific, Inc.). The amplification was performed according to the following conditions: Denaturation at $95^{\circ} \mathrm{C}$ for $15 \mathrm{sec}$, and 40 cycles of annealing at $60^{\circ} \mathrm{C}$ for $45 \mathrm{sec}$ and extending at $72^{\circ} \mathrm{C}$ for $30 \mathrm{sec}$. The primers used were as follows: E-cadherin forward, 5'-TGCTGCAGG TCTCCTCTTGG-3' and reverse, 5'-AGTCCCAGGCGTAGA CCAAG-3'; Sirt7 forward, 5'-TACATTGAAGTCTGTACC TCC-3' and reverse, 5'-GTGGGTACTTCTTTAGAACCT-3'; Vimentin forward 5'-ATTGAGATTGCCACCTACAG-3' and reverse 5'-ATCCAGATTAGTTTCCCTCAG-3'; GAPDH, forward 5'-GAGAAGTATGACAACAGCCTC-3' and reverse 5'-ATGGACTGTGGTCATGAGTC-3'. The miRNA levels were normalized against GAPDH and relative fold changes were calculated using the $2^{-\Delta \Delta \mathrm{Cq}}$ method (15).

Western blot analysis. Cells were lysed using radioimmunoprecipitation assay buffer at $4^{\circ} \mathrm{C}$ for $45 \mathrm{~min}$, and the protein concentration was determined using a bicinchoninic acid assay kit (Thermo Fisher Scientific, Inc.). Next, $30 \mu \mathrm{g}$ protein was resolved with 10\% SDS-polyacrylamide gel electrophoresis and transferred to a polyvinylidene difluoride membrane (EMD Millipore, Billerica, MA, USA). Following blocking with WB blocking solution (Beyotime Institute of Biotechnology, Zhejiang, China), the membrane was incubated overnight at $4^{\circ} \mathrm{C}$ with primary antibodies. The primary antibodies used were from an epithelial-mesenchymal transition (EMT) antibody kit (cat. no. 9782; Cell Signaling Technology, Inc., Danvers, MA, USA) in which each antibody was diluted at 1:1,000, the SIRT7 antibody (cat. no. ab62748; 1:500; Abcam, Cambridge, MA, USA) and the GAPDH antibody (cat. no. SC81545; 1:1,000; Santa Cruz Biotechnology, Inc., Dallas, TX, USA) was used as an internal control. Following three washes with extensive Tris-buffered saline/Tween-20 (TBST) for $10 \mathrm{~min}$, peroxidase-conjugated goat anti-rabbit (sc-2004; 1:3,000) or goat anti-mouse IgG secondary antibodies (sc-2005; 1:3,000) (both Santa Cruz Biotechnology, Inc.) were used for incubation at room temperature for $1 \mathrm{~h}$. Following the washing of the membrane with TBST four times for $15 \mathrm{~min}$, the immunoreactivity was detected using an enhanced chemiluminescence kit (Pierce; Thermo Fisher Scientific, Inc.) and quantified using ImageJ software (version 2.1.4; National Institutes of Health, Bethesda, MD, USA). 
Table I. Clinicopathological variables in patients with colorectal carcinoma.

\begin{tabular}{|c|c|c|c|c|}
\hline \multirow[b]{2}{*}{ Characteristics } & \multirow[b]{2}{*}{ No. } & \multicolumn{2}{|c|}{ Expression of Sirt7 } & \multirow[b]{2}{*}{ P-value } \\
\hline & & Low $(\mathrm{n}=21)$ & High $(n=39)$ & \\
\hline Gender & & & & 0.183 \\
\hline Male & 27 & 7 & 20 & \\
\hline Female & 33 & 14 & 19 & \\
\hline Age, years & & & & 0.765 \\
\hline$<50$ & 27 & 10 & 17 & \\
\hline$\geq 50$ & 33 & 11 & 22 & \\
\hline Tumor size (diameter) & & & & 0.009 \\
\hline Small $(\leq 3 \mathrm{~cm})$ & 29 & 15 & 14 & \\
\hline Large $(\geq 3 \mathrm{~cm})$ & 31 & 6 & 25 & \\
\hline Tumor, node and metastasis stage & & & & 0.003 \\
\hline $\mathrm{I}-\mathrm{II}$ & 30 & 16 & 14 & \\
\hline III-IV & 30 & 5 & 25 & \\
\hline Lymph node metastasis & & & & 0.123 \\
\hline Absent & 29 & 13 & 16 & \\
\hline Present & 31 & 8 & 23 & \\
\hline Distant metastasis & & & & 0.016 \\
\hline Absent & 22 & 12 & 10 & \\
\hline Present & 38 & 9 & 29 & \\
\hline Tumor location & & & & 0.417 \\
\hline Colon & 30 & 12 & 18 & \\
\hline Rectum & 30 & 9 & 21 & \\
\hline
\end{tabular}

Cell proliferation assay. MTT and colony formation assays were performed to measure the cell growth viability. For MTT assay, the transfected cells were seeded into 96-well plate in triplicate, at a concentration of 500 cells/well. At 24, 48, 72 and $96 \mathrm{~h}$ after transfection, $20 \mu \mathrm{MTT}(5 \mathrm{mg} / \mathrm{ml})$ was added to each well and further incubated for $4 \mathrm{~h}$ at $37^{\circ} \mathrm{C}$, followed by addition of $150 \mu \mathrm{l}$ of dimethyl sulfoxide to stop the reaction. The absorbance of each well was measured at the wavelength of $570 \mathrm{~nm}$ on a microplate reader in three independent experiments.

For the colony formation assay, the transfected cells were seeded into 6-well plates in triplicate at a density of $1 \times 10^{3}$ cells/well and cultured for 10 days at $37^{\circ} \mathrm{C}$. Subsequent to fixing with $10 \%$ paraformaldehyde for $15 \mathrm{~min}$ at room temperature, the colonies were stained with Giemsa for $30 \mathrm{~min}$ at room temperature. Colonies with $>50$ cells were counted and analyzed.

Cell invasion analysis. For the invasion assay, Transwell chambers precoated with Matrigel were obtained from BD Biosciences (San Jose, CA, USA). Transfected cells ( $2 \times 10^{4}$ cells per well) in RPMI-1640 medium were added to the upper chambers. RPMI-1640 with $10 \%$ FBS was added to the lower chambers. At $24 \mathrm{~h}$ after transfection, non-migrating cells on the upper side were gently wiped off, while the cells that migrated through the filter were fixed with $4 \%$ polyoxymethylene for $20 \mathrm{~min}$ at room temperature, stained with $1 \%$ crystal violet for $30 \mathrm{~min}$ at room temperature
(Sigma-Aldrich; Merck KGaA) and counted using phase-contrast microscopy.

Luciferase reporter assay. The luciferase reporter activity was conducted using a Luciferase Assay system (Promega Corporation, Madison, WI, USA). E-cadherin (-108)-Luc and Mutant E-cadherin (-108)-Luc were generated as described previously (16). By transfecting the E-cadherin reporter construct and Sirt7 or siSirt7 into the indicated cell lines, and co-transfecting with pRL-SV40 renilla luciferase vector as an internal control for transfection efficiency, luciferase reporter activity was measured. Cells were harvested after $48 \mathrm{~h}$ and lysates were assayed for luciferase activity, according to the manufacturer's protocol. Luciferase activities were normalized to renilla luciferase activity. Each experiment was performed in triplicate.

Statistical analysis. All statistical analyses were performed using SPSS version 17.0 software (SPSS, Inc., Chicago, IL, USA). Each experiment was performed for at least three independent times and the data were presented as the means \pm standard deviation. Differences were analyzed using $\chi^{2}$ test, Student's t-test or one-way analysis of variance accordingly. Expression comparisons between two groups were performed with a Student's t-test. The $\chi^{2}$ test was used to evaluate the association between Sirt7 expression and the clinicopathological characteristics of patients. Cox log-rank test was used to test the prognostic significance. $\mathrm{P}<0.05$ 

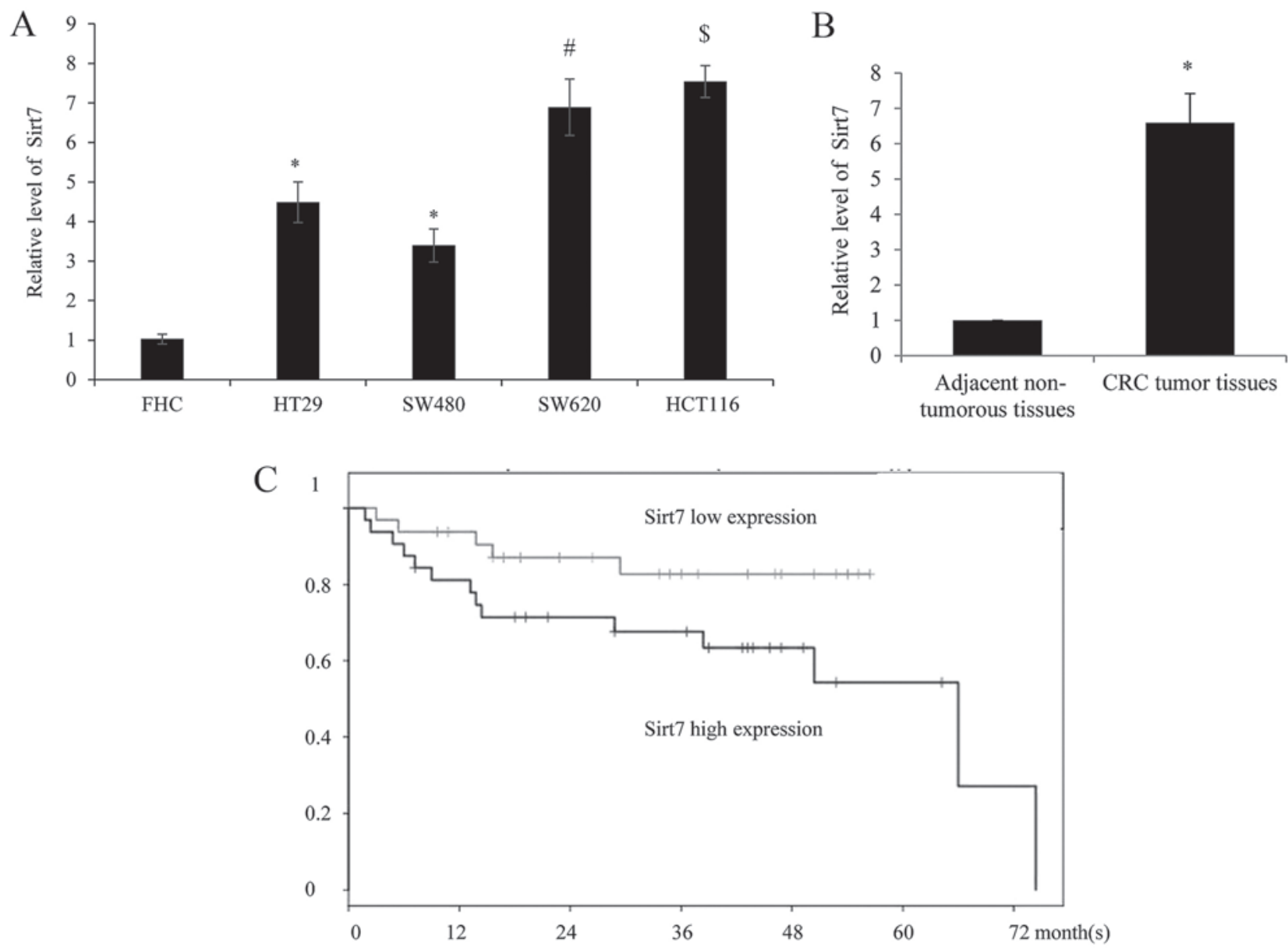

Figure 1. Sirt7 expression was upregulated in CRC cell lines and tissues. Reverse transcription-quantitative polymerase chain reaction was used to investigate the Sirt7 mRNA expression in (A) four CRC cell lines (HT29, SW480, SW620 and HCT116) and normal colorectal FHC cell lines, as well as in (B) 60 paired CRC tissues and adjacent normal tissues. GAPDH served as an internal control. The data are presented as the mean \pm standard deviation of three independent experiments. ${ }^{~} \mathrm{P}<0.05$ vs. control FHC cells or tissues. ${ }^{~} \mathrm{P}<0.05$ vs. HT2 2 cells. ${ }^{5} \mathrm{P}<0.05$ vs. SW480 cells. (C) Kaplan-Meier curves were used to measure the patient survival rate according to Sirt7 expression level, all patients in the low expression group succumbed prior to the 72 month follow up. Cox log-rank test was used to test the prognostic significance. Sirt7, sirtuin 7; CRC, colorectal carcinoma.

was considered as an indicator of a statistically significant difference.

\section{Results}

Expression of Sirt7 is upregulated in CRC cell lines and tissues. In order to examine the expression level of Sirt7 in different CRC cell lines (HT29, SW480, SW620 and HCT116), along with the human normal colorectal cell line FHC, the mRNA of cells was harvested and analyzed by RT-qPCR. The results identified that Sirt7 exhibited a significantly higher expression level in the CRC cells as compared with the normal FHC cells (Fig. 1A). Furthermore, compared with the low-metastatic tumor cells HT29 and SW480, a higher expression of Sirt7 was detected in the highly-metastatic SW620 and HCT116 cells, respectively.

RT-qPCR was also used to assess the expression of Sirt7 in $60 \mathrm{CRC}$ and adjacent non-tumorous tissues. As shown in Fig. 1B, Sirt7 was significantly upregulated in CRC tumor tissues compared with the corresponding normal tissues. The clinical information of Sirt7 expression is summarized in Table I, which indicates that higher expression of Sirt7 was correlated with the tumor size, TNM stage and distant metastasis in patients. However, there was no statistically significant difference between the age, gender, lymph node metastasis and tumor location, and the expression of Sirt7. Furthermore, the association between Sirt7 expression and patient survival times was investigated. Depending on the median Sirt7 expression level, the patients were divided into the high (relative expression 22.57 ) and low (relative expression <2.57) expression groups. Higher Sirt7 expression was correlated with a worse overall survival rate, according to the Kaplan-Meier curves, however all patients in the low expression group succumbed prior to the 72 month follow-up (Fig. 1C).

Sirt7 exhibits oncogenic properties by promoting CRC cell proliferation. Since higher expression of Sirt7 was found to be correlated with tumor size, it was hypothesized that Sirt7 may promote CRC cell proliferation. In order to examine the role of Sirt7, an RNA interference assay was performed to silence the expression of Sirt7 (si-Sirt7 transfected group) in SW620 and HCT116 cells. The transfection efficiency was analyzed using RT-qPCR, and knockdown of Sirt7 was observed in the transfected cells (Fig. 2A). Furthermore, the MTT assay in the two cell lines indicated that silencing of Sirt7 may inhibit 

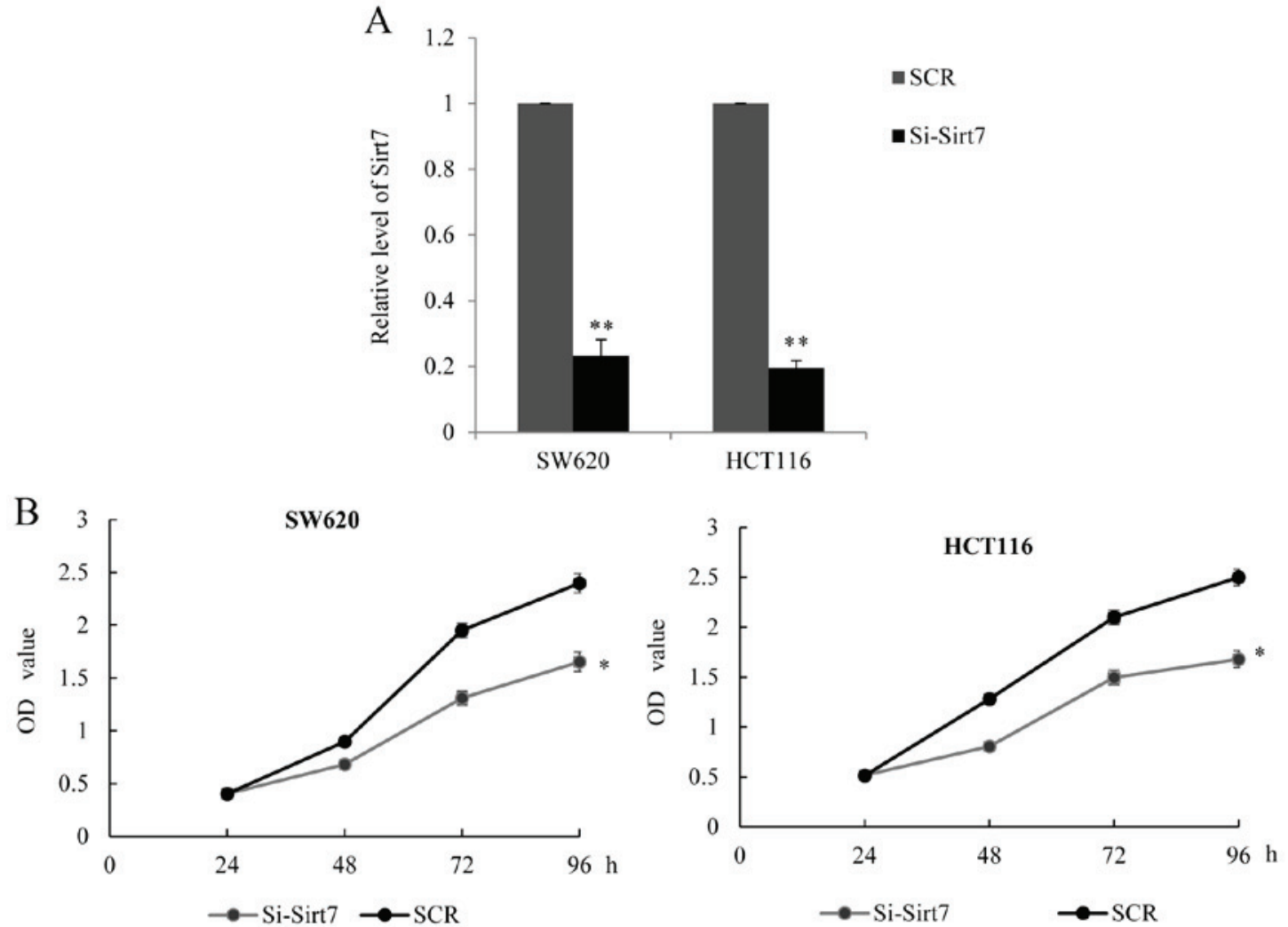

C

SW620

\section{HCT116}
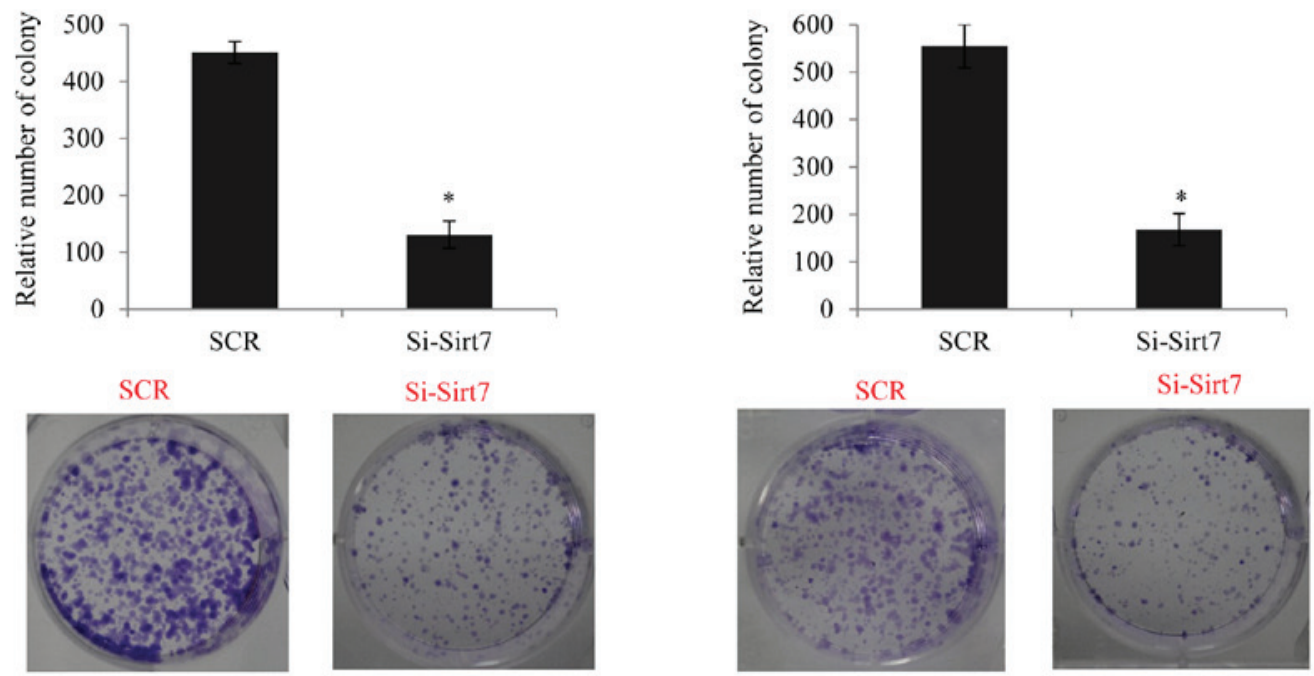

Figure 2. Sirt7 exhibited oncogenic properties by promoting CRC cell proliferation. (A) Endogenous Sirt7 level was measured in SW620 and HCT116 cells following transduction with corresponding Sirt7 siRNAs or SCR (negative control). GAPDH served as an internal control for each group. (B) MTT assay was performed in SW620 and HCT116 cells transfected with si-Sirt7 or SCR. The OD value was measured every $24 \mathrm{~h}$, demonstrating that knockdown of Sirt7 significantly reduced CRC proliferation. (C) Colony formation number was counted and analyzed in SW620 and HCT116 cells transfected with si-Sirt7 or SCR. "P<0.05 and ${ }^{* *} \mathrm{P}<0.01$ vs. corresponding control group. Sirt7, sirtuin 7; CRC, colorectal carcinoma; SCR, scramble control RNA; OD, optical density; si, siRNA.

cancer cell proliferation (Fig. 2B). The colony formation assay also revealed that the Sirt7 knockdown group had a lower colony-forming efficiency in comparison with the control SCR group (Fig. 2C). The aforementioned data confirmed that a decreased expression of Sirt7 may have a suppressive effect on CRC cell proliferation.

Overexpression of Sirt7 increases the invasion ability of CRC cells. To investigate whether Sirt7 was involved in the CRC invasion, a Sirt7-overexpression lentivirus was transfected into HT29 and SW480 cells, which presented lower metastatic ability and Sirt7 expression compared with the SW620 and HCT116 cells, as observed earlier.

The efficiency of Sirt7 overexpression was assessed by RT-qPCR. Empty lentiviral vector GV208, which did not contain any external sequence, was used as the negative control (pGV-NC). As shown in Fig. 3A, significant overexpression of Sirt7 was observed in the lentivirus transfected 


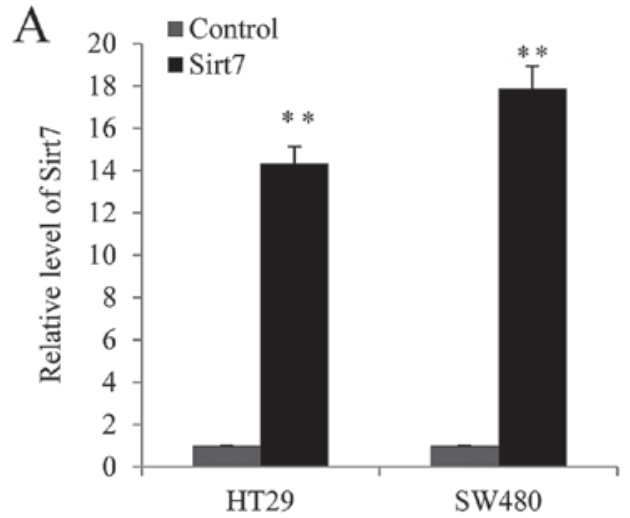

B

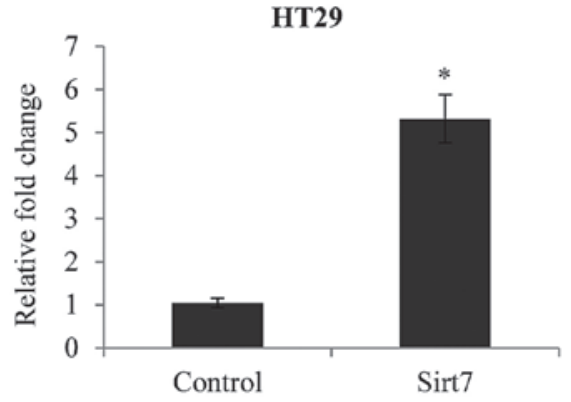

$\mathrm{C}$

范

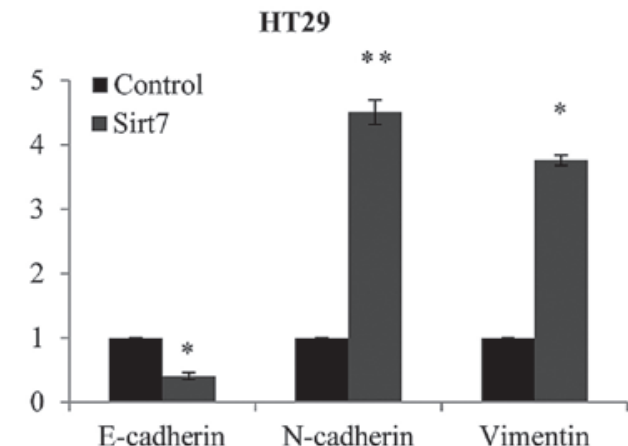

$\mathrm{D}$

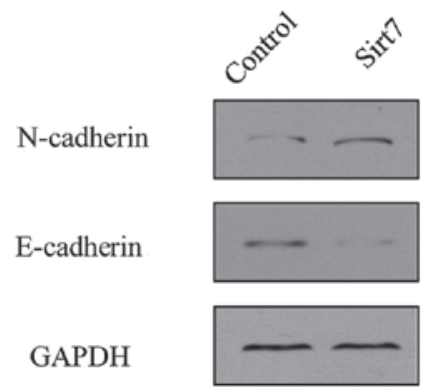

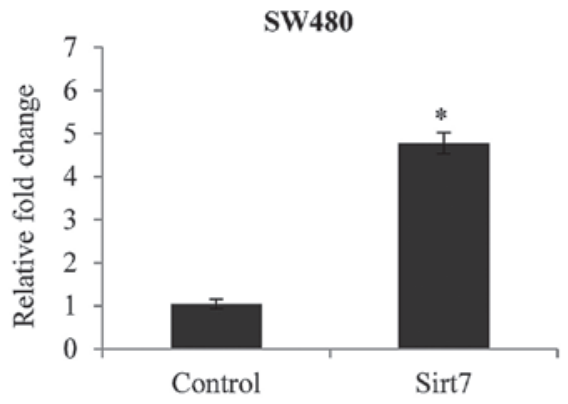

SW480

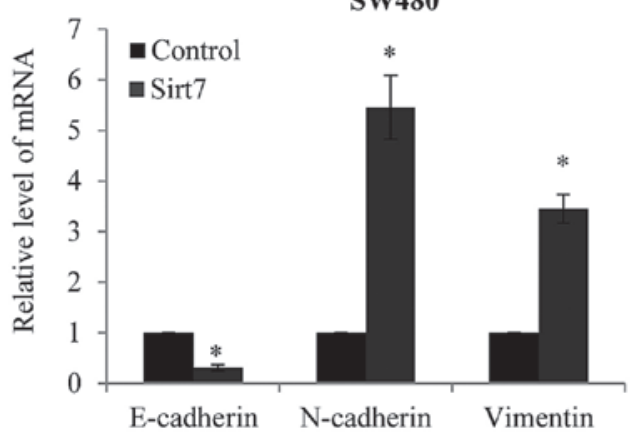

$\mathrm{N}$-cadherin

E-cadherin

GAPDH
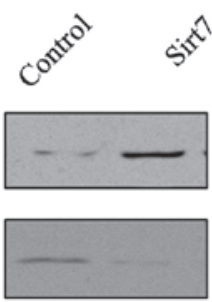

si

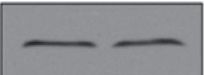

Figure 3. Overexpression of Sirt7 increased the invasion ability of CRC cells. (A) Endogenous Sirt7 level was measured in HT29 and SW480 cells following transduction with Sirt7 overexpression lentivirus or a lentiviral GV208 vector (control). GAPDH served as an internal control. (B) Transwell assay was performed in the HT29 and SW480 cells transfected with Sirt7 overexpression lentivirus or control vector. The cells invading the lower chamber were stained and counted under a light microscopy, and the results are presented as the fold change over the control group. (C) Reverse transcription-quantitative polymerase chain reaction analysis demonstrated the expression levels of the epithelial-mesenchymal transition markers (E-cadherin, $\mathrm{N}$-cadherin and vimentin) following overexpression of Sirt7. (D) Western blot analysis was used to detect the changes in E-cadherin and N-cadherin following overexpression of Sirt7. GAPDH was used as a control. ${ }^{*} \mathrm{P}<0.05$ and ${ }^{* *} \mathrm{P}<0.01$, vs. corresponding control group. Sirt7, sirtuin 7; CRC, colorectal carcinoma; SCR, scramble control RNA.

cells. Furthermore, a Transwell assay was performed in the HT29 and SW480 cells, and cells transfected with the Sirt7 overexpression vector exhibited a significantly increased ability of invasion in the two cell lines (Fig. 3B).

As EMT is usually considered as the initial step of metastasis, the present study detected the changes in the EMT markers, E-cadherin, N-cadherin and vimentin. In HT29 and
SW480 cells with overexpression of Sirt7, a marked upregulation of $\mathrm{N}$-cadherin and vimentin mRNA expression was observed, as well as significant downregulation of E-cadherin (Fig. 3C). The changes in the expression levels of these markers were also detected by western blot analysis; however, since the antibody for vimentin is not available, western blotting was not performed for vimentin. As shown in Fig. 3D, 
A

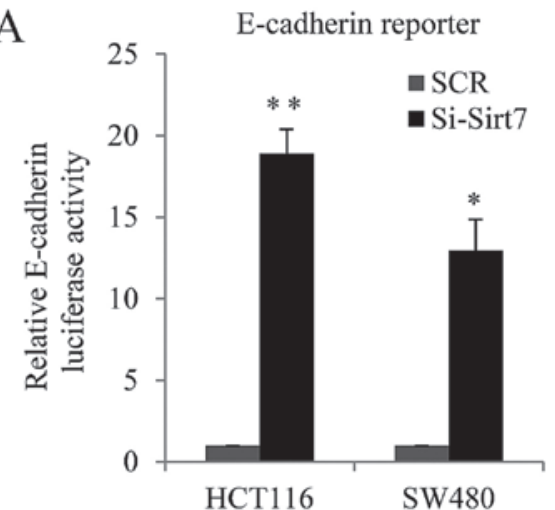

$\mathrm{C}$

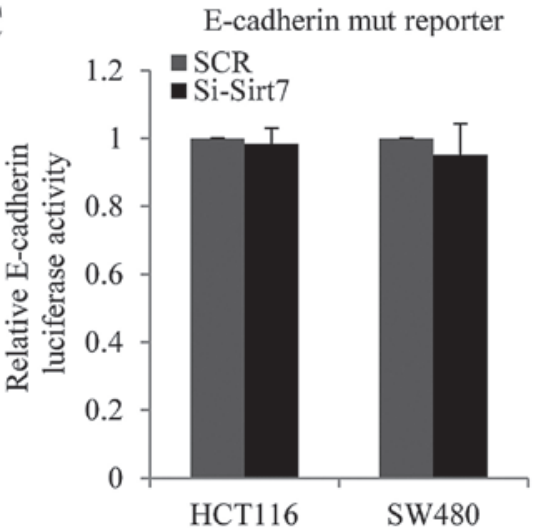

$\mathrm{B}$ E-cadherin reporter

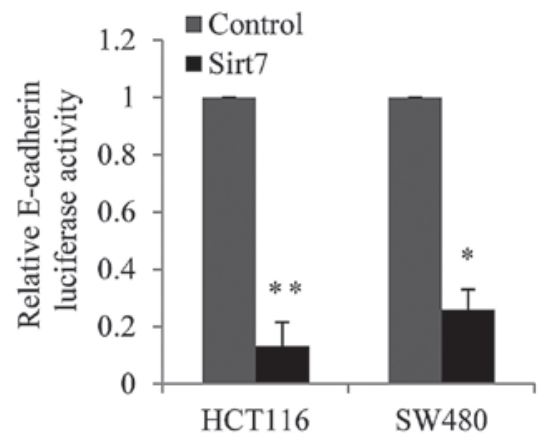

$\mathrm{D}$ E-cadherin mut reporter

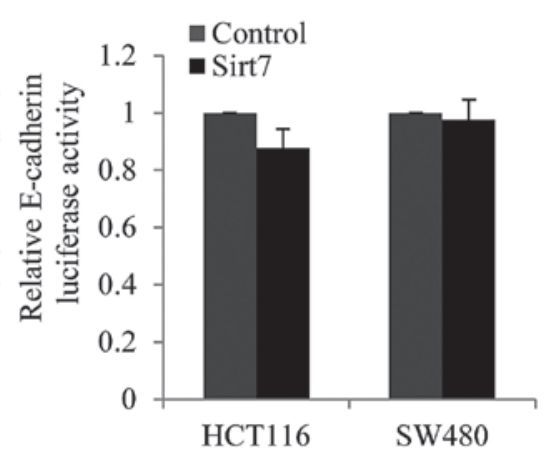

Figure 4. Sirt7 regulated E-cadherin transcription in an E-box-dependent manner. Relative E-cadherin luciferase activity was measured in HCT116 or SW480 cells co-transfected with: (A) Sirt7 siRNAs (for Sirt7 knockdown) or SCR, along with E-cadherin luciferase reporter vector (pGL-E-cadherin) and b-gal constructs; (B) Sirt7-overexpression lentivirus or vector lentivirus, along with E-cadherin luciferase reporter vector (pGL-E-cadherin) and b-gal constructs; (C) Sirt7 siRNAs or SCR along with the E-box-mutated E-cadherin luciferase reporter vector (pGL-E-box-mut) and b-gal constructs; (D) Sirt7-overexpression lentivirus or vector lentivirus, along with the E-box-mutated E-cadherin luciferase reporter vector (pGL-E-box-mut) and b-gal constructs. In order to control for transfection efficiency, the relative luciferase activities were normalized to the b-gal activity. Each experiment was performed in triplicate. The error bars represent the mean \pm standard deviation. ${ }^{*} \mathrm{P}<0.05$ and ${ }^{* *} \mathrm{P}<0.01$, vs. corresponding control group. Sirt7, sirtuin 7; CRC, colorectal carcinoma; SCR, scramble control RNA; si, siRNA.

increase of $\mathrm{N}$-cadherin and decrease of E-cadherin protein levels were observed following Sirt7 overexpression. These findings supported the theory that Sirt7 expression enhanced CRC EMT and invasion.

Sirt7 regulates $E$-cadherin transcription in an E-box-dependent manner. It is known that the overexpression of Sirt1 stimulates cell invasion by suppressing E-cadherin expression in various cancer types $(17,18)$. Thus, in the present study, it was hypothesized that Sirt7, a new Sirt family member, may also regulate E-cadherin. Luciferase assay was performed to examine the function of Sirt7. An E-cadherin luciferase-reporter construct was co-transfected along with si-Sirt7 (knockdown) or Sirt7-overexpression vector and their corresponding controls into HCT116 and SW480 cells. The results revealed that E-cadherin luciferase activity was increased in the Sirt7-knockdown cells as compared with the SCR cells (Fig. 4A). By contrast, when Sirt7 was overexpressed in the two cell lines, the E-cadherin luciferase activity was decreased (Fig. 4B). Furthermore, E-box domain mutation of E-cadherin was investigated in order to confirm whether the inhibition of E-cadherin expression by Sirt7 was dependent on the inhibition of the E-box.
Subsequently, co-transfection with si-Sirt7 and E-box-mutated E-cadherin luciferase reporters was conducted in HCT116 or SW480 cells, and the luciferase report activity was measured. As shown in Fig. 4C, the knockdown of Sirt7 had almost no effect on the E-box-mutated E-cadherin luciferase reporter. HCT116 or SW480 cells were also co-transfected with Sirt7-overexpression lentivirus and E-box-mutated E-cadherin luciferase reporters in HCT116 cells or SW480 cells. As shown from Fig. 4D, Sirt7 exerted a reduced effect on the E-box-mutated E-cadherin promoter compared with the E-box wild type promoter. These results demonstrated that Sirt7 suppressed E-cadherin expression at the transcriptional level in an E-box-dependent manner in the CRC cell lines.

Sirt7 regulates $C R C$ proliferation and invasion in an E-cadherin-dependent manner. Based on the aforementioned results, the present study further investigated whether Sirt7 regulates CRC proliferation and metastasis in an E-cadherin-dependent manner. RNA interference targeting the CDH1 gene, which encodes the E-cadherin protein, was performed to silence the expression of E-cadherin in SW620 and HCT116 cells. The transfection efficiency was analyzed using RT-qPCR (Fig. 5A). In SW620 and HCT116 cells, 
A

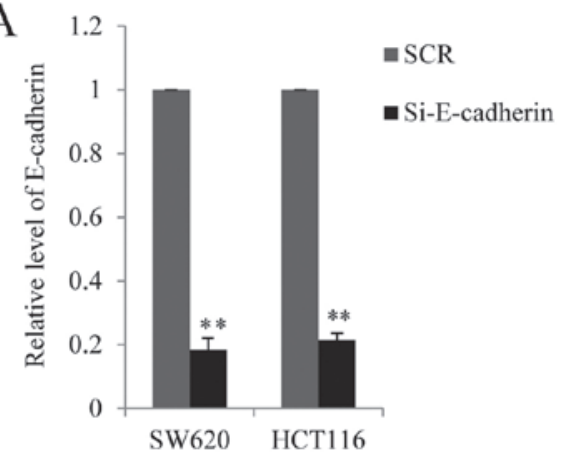

$\mathrm{C}$

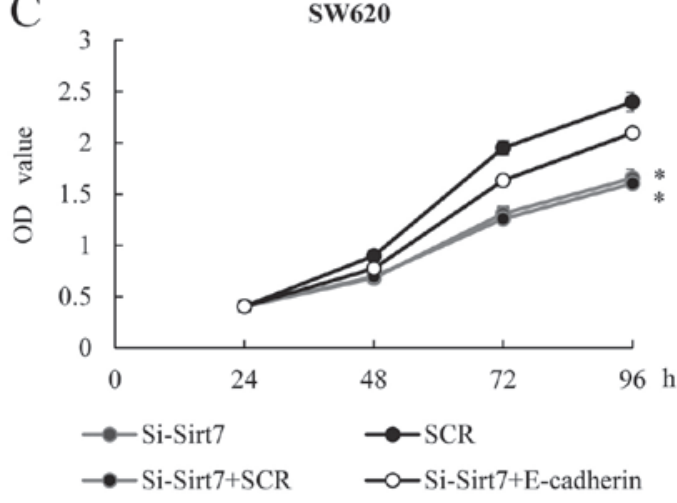

$\mathrm{D}$

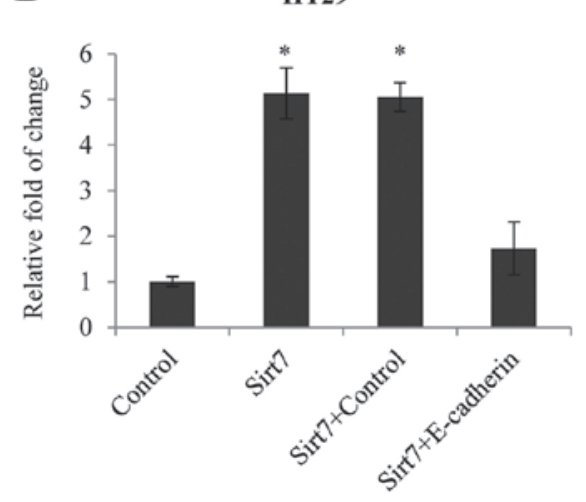

B
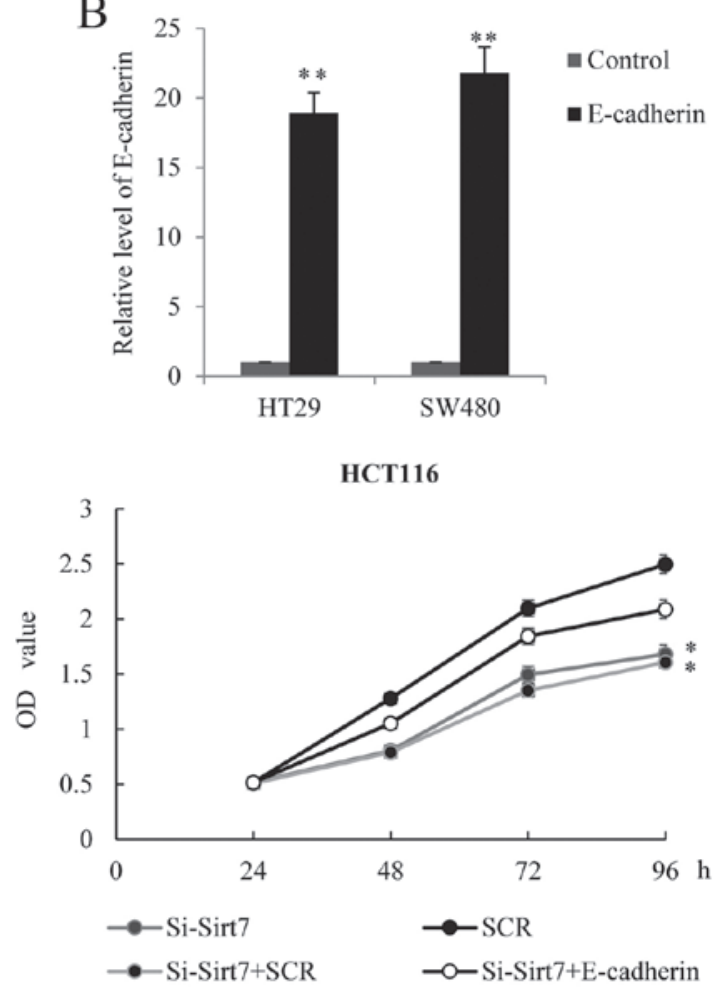

SW480

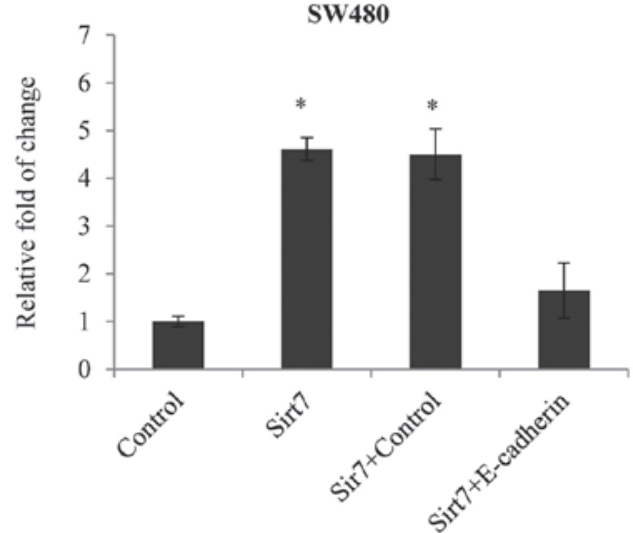

Figure 5. Sirt7 regulated CRC proliferation and invasion in an E-cadherin-dependent manner. Endogenous E-cadherin level was measured in SW620 and HCT116 cells after transduction with (A) E-cadherin siRNAs or SCR and (B) E-cadherin overexpression lentivirus or lentiviral vector GV208 (control). GAPDH served as an internal control. (C) MTT assay was performed in SW620 and HCT116 cells transfected with si-Sirt7, SCR, si-Sirt7+SCR or si-Sirt7+si-E-cadherin. The OD value was measured every $24 \mathrm{~h}$. (D) Transwell assay was performed in HT29 and SW480 cells transfected with Sirt7, vector, Sirt7+vector or Sirt7+E-cadherin. Cells invading the lower chamber were stained and counted under a light microscopy, and the results were presented as the fold change over the vector. ${ }^{*} \mathrm{P}<0.05$ and ${ }^{* *} \mathrm{P}<0.01$, vs. corresponding control group. Sirt7, sirtuin 7; CRC, colorectal carcinoma; SCR, scramble control RNA; si, siRNA.

E-cadherin was overexpressed using a lentivirus and the efficiency of E-cadherin overexpression was assessed by RT-qPCR, with an empty lentiviral GV208 vector used as a control (Fig. 5B). The data demonstrated that the siRNA and the E-cadherin overexpression lentiviruses were successful and thus, were used for the analysis of Sirt7 function. The MTT assay in the SW620 and HCT116 cells indicated that the effect of Sirt7 knockdown on proliferation may be partially reduced by the E-cadherin knockdown (Fig. 5C). A Transwell assay in the HT29 and SW480 cells also demonstrated that, while E-cadherin was overexpressed in the cells transfected with Sirt7-overexpression constructs, the invasion ability was reduced (Fig. 5D). On the basis of these results, it can be concluded that Sirt7 regulated CRC proliferation and invasion in an E-cadherin-dependent manner.

\section{Discussion}

Metastasis is one of the main hallmarks of cancer and the leading cause of cancer-associated mortality (19). Epigenetic aberrations have been demonstrated to contribute to the process of tumorigenesis and metastasis in various ways (20). Therefore, investigating the epigenetic regulation of CRC may provide new insight into the underlying molecular mechanisms and thus assist in the development of novel clinically relevant prognostic biomarkers. 
The Sirt family members target numerous key proteins to modulate their state, from acetylation to deacetylation, and have been reported to be involved in several pathological conditions, including malignant tumors, cardiovascular disease and diabetes (21-24). The function of Sirtl in CRC has been well discussed, and high Sirtl expression has been reported to enhance tumorigenesis and be associated with a poor prognosis of CRC patients, including advanced-stage tumors and lymph node or liver metastases $(25,26)$. By targeting fos-related antigen 1, Sirt1 promotes EMT and metastasis in CRC (27). While numerous studies on Sirt1 have examined its biological properties, the expression panel and function of Sirt7, a new member of the Sirt family, in human malignancies remains unclear.

In recent years, emerging studies have been examined the underlying mechanism of Sirt7 in promoting cancer development, including hepatocellular carcinoma (28), bladder cancer (29) and ovarian cancer (4). Yu et al (30) reported that Sirt7 expression was elevated in CRC tissues and cells, and was correlated with tumor stage and poor prognosis. In Sirt7-overexpressing cells, vimentin and fibronectin were upregulated, whereas E-cadherin and $\beta$-catenin were downregulated (20), which was consistent with the results of the present study; however, the aforementioned study did not reveal the reasons underlying these observations. In the present study, the initial aim was to uncover the mechanism underlying the Sirt7 enhancing effect on CRC cell proliferation and invasion, and shows evidence of the potential rationale for Sirt7 as a therapeutic target. The current results highlight that Sirt7 not only increased $\mathrm{N}$-cadherin expression, but also regulated $\mathrm{E}$-cadherin transcription in an E-box-dependent manner. Notably, although E-cadherin is traditionally considered as a suppressor of cell invasion, the present study observed the inhibitory function of E-cadherin on the cell proliferation, similarly to the findings of Kim et al (31) and Ji et al (32) reported in CRC and pancreatic cancer. Although the higher Sirt7 group had a worse overall survival rate, the deaths of the lower Sirt7 group may be due to the complicated tumor metastatic mechanism. Sirt7 is not the only oncogene for CRC.

In conclusion, the present study demonstrated that Sirt7 may serve as an oncogene and therapeutic target in patients with CRC by directly inhibiting the expression of E-cadherin. However, further studies are required to better define the underlying molecular mechanisms and to explore the use of Sirt7 inhibitors in CRC. In addition, the function of Sirt7 in migration should be further studied, as the migration activity was not analyzed in the current study. Finally, a larger independent CRC patient cohort is also necessary for the validation of these results.

\section{References}

1. Kelly G: A review of the sirtuin system, its clinical implications, and the potential role of dietary activators like resveratrol: part 1. Altern Med Rev 15: 245-263, 2010.

2. Kelly GS: A review of the sirtuin system, its clinical implications, and the potential role of dietary activators like resveratrol: part 2. Altern Med Rev 15: 313-328, 2010.

3. Barber MF, Michishita-Kioi E, Xi Y, Tasselli L, Kioi M, Moqtaderi Z, Tennen RI, Paredes S, Young NL, Chen K, et al: SIRT7 links H3K18 deacetylation to maintenance of oncogenic transformation. Nature 487: 114-118, 2012.
4. Wang HL, Lu RQ, Xie SH, Zheng H, Wen XM, Gao X and Guo L: SIRT7 Exhibits oncogenic potential in human ovarian cancer cells. Asian Pac J Cancer Prev 16: 3573-3577, 2015.

5. Zhang S, Chen P, Huang Z, Hu X, Chen M, Hu S, Hu Y and Cai T: Sirt7 promotes gastric cancer growth and inhibits apoptosis by epigenetically inhibiting miR-34a. Sci Rep 5: 9787, 2015.

6. Geng Q, Peng H, Chen F, Luo R and Li R: High expression of Sirt7 served as a predictor of adverse outcome in breast cancer. Int J Clin Exp Pathol 8: 1938-1945, 2015.

7. Singh S, Kumar PU, Thakur S, Kiran S, Sen B Sharma S, Rao VV, Poongothai AR and Ramakrishna G: Expression/localization patterns of sirtuins (SIRT1, SIRT2, and SIRT7) during progression of cervical cancer and effects of sirtuin inhibitors on growth of cervical cancer cells. Tumour Biol 36: 6159-6171, 2015.

8. Torre LA, Bray F, Siegel RL, Ferlay J, Lortet-Tieulent J and Jemal A: Global cancer statistics, 2012. CA Cancer J Clin 65: 87-108, 2015.

9. Brenner H, Kloor M and Pox CP: Colorectal cancer. Lancet 383: 1490-1502, 2014

10. Ferrarotto R, Pathak P, Maru D, Agarwal A, Overman M, Hoff PM and Kopetz S: Durable complete responses in metastatic colorectal cancer treated with chemotherapy alone. Clin Colorectal Cancer 10: 178-182, 2011.

11. Kraus S, Nabiochtchikov I, Shapira S and Arber N: Recent advances in personalized colorectal cancer research. Cancer Lett 347: 15-21, 2014.

12. Park IJ and Yu CS: Current issues in locally advanced colorectal cancer treated by preoperative chemoradiotherapy. World J Gastroenterol 20: 2023-2029, 2014.

13. Andre $\mathrm{N}$ and Schmiegel W: Chemoradiotherapy for colorectal cancer. Gut 54: 1194-1202, 2005.

14. O'Connell JB, Maggard MA and Ko CY: Colon cancer survival rates with the new American Joint Committee on Cancer sixth edition staging. J Natl Cancer Inst 96: 1420-1425, 2004.

15. Livak KJ and Schmittgen TD: Analysis of relative gene expression data using real-time quantitative PCR and the 2(-Delta Delta C(T)) method. Methods 25: 402-408, 2001.

16. Hajra KM, Chen DY and Fearon ER: The SLUG zinc-finger protein represses E-cadherin in breast cancer. Cancer Res 62: 1613-1618, 2002.

17. Deng S, Zhu S, Wang B, Li X, Liu Y, Qin Q, Gong Q, Niu Y, Xiang C, Chen J, et al: Chronic pancreatitis and pancreatic cancer demonstrate active epithelial-mesenchymal transition profile, regulated by miR-217-SIRT1 pathway. Cancer Lett 355: 184-191, 2014.

18. Byles V, Zhu L, Lovaas JD, Chmilewski LK, Wang J, Faller DV and Dai Y: SIRT1 induces EMT by cooperating with EMT transcription factors and enhances prostate cancer cell migration and metastasis. Oncogene 31: 4619-4629, 2012.

19. Hanahan D and Weinberg RA: Hallmarks of cancer: The next generation. Cell 144: 646-674, 2011.

20. Ellis L, Atadja PW and Johnstone RW: Epigenetics in cancer: Targeting chromatin modifications. Mol Cancer Ther 8: 1409-1420, 2009.

21. Ashraf N, Zino S, Macintyre A, Kingsmore D, Payne AP, George WD and Shiels PG: Altered sirtuin expression is associated with node-positive breast cancer. Br J Cancer 95: 1056-1061, 2006.

22. Zhang L, Ren X, Cheng Y, Huber-Keener K, Liu X, Zhang Y, Yuan YS, Yang JW, Liu CG and Yang JM: Identification of Sirtuin 3, a mitochondrial protein deacetylase, as a new contributor to tamoxifen resistance in breast cancer cells. Biochem Pharmacol 86: 726-733, 2013.

23. Dong XC: Sirtuin biology and relevance to diabetes treatment. Diabetes Manag (Lond) 2: 243-257, 2012.

24. Calabrese V, Cornelius C, Leso V, Trovato-Salinaro A, Ventimiglia B, Cavallaro M, Scuto M, Rizza S, Zanoli L, Neri S and Castellino P: Oxidative stress, glutathione status, sirtuin and cellular stress response in type 2 diabetes. Biochim Biophys Acta 1822: 729-736, 2012.

25. Chen X, Sun K, Jiao S, Cai N, Zhao X, Zou H, Xie Y, Wang Z, Zhong M and Wei L: High levels of SIRT1 expression enhance tumorigenesis and associate with a poor prognosis of colorectal carcinoma patients. Sci Rep 4: 7481, 2014.

26. Lv L, Shen Z, Zhang J, Zhang H, Dong J, Yan Y, Liu F, Jiang K, Ye Y and Wang S: Clinicopathological significance of SIRT1 expression in colorectal adenocarcinoma. Med Oncol 31: 965, 2014. 
27. Cheng F, Su L, Yao C, Liu L, Shen J, Liu C, Chen X, Luo Y, Jiang L, Shan J, et al: SIRT1 promotes epithelial-mesenchymal transition and metastasis in colorectal cancer by regulating Fra-1 expression. Cancer Lett 375: 274-283, 2016.

28. Kim JK, Noh JH, Jung KH, Eun JW, Bae HJ, Kim MG, Chang YG, Shen Q, Park WS, Lee JY, et al: Sirtuin7 oncogenic potential in human hepatocellular carcinoma and its regulation by the tumor suppressors MiR-125a-5p and MiR-125b. Hepatology 57: 1055-1067, 2013.

29. Han Y, Liu Y, Zhang H, Wang T, Diao R, Jiang Z, Gui Y and Cai Z: Hsa-miR-125b suppresses bladder cancer development by down-regulating oncogene SIRT7 and oncogenic long non-coding RNA MALAT1. FEBS Lett 587: 3875-3882, 2013.

30. Yu H, Ye W, Wu J, Meng X, Liu RY, Ying X, Zhou Y, Wang H, Pan $\mathrm{C}$ and Huang W: Overexpression of sirt7 exhibits oncogenic property and serves as a prognostic factor in colorectal cancer. Clin Cancer Res 20: 3434-3445, 2014.
31. Kim S, Ahn SH, Yang HY, Lee JS, Choi HG, Park YK and Lee TH: Modification of cysteine 457 in plakoglobin modulates the proliferation and migration of colorectal cancer cells by altering binding to E-cadherin/catenins. Redox Rep 22: 272-281, 2017.

32. Ji M, Fan D, Yuan L, Zhang Y, Dong W and Peng X: EBP50 inhibits pancreatic cancer cell growth and invasion by targeting the beta-catenin/E-cadherin pathway. Exp Ther Med 10: 1311-1316, 2015.

This work is licensed under a Creative Commons Attribution-NonCommercial-NoDerivatives 4.0 International (CC BY-NC-ND 4.0) License. 